\title{
Phonons and Electron Correlations in High-Temperature and Other Novel Superconductors
}

\author{
Alexandre Sasha Alexandrov, ${ }^{1}$ Carlo Di Castro, ${ }^{2}$ Igor Mazin, ${ }^{3}$ and Dragan Mihailovic ${ }^{4}$ \\ ${ }^{1}$ Department of Physics, Loughborough University, Loughborough LE11 3TU, UK \\ ${ }^{2}$ Dipartimento di Fisica, Università degli Studi di Roma "La Sapienza", Piazzale Aldo Moro 5, 00185 Roma, Italy \\ ${ }^{3}$ U.S. Naval Research Laboratory, code 6390, 4555 Overlook Avenu SW, Washington, DC 20375, USA \\ ${ }^{4}$ Department of Complex Matter, Jozef Stefan Institute, Ljubljana 1001, Slovenia \\ Correspondence should be addressed to Alexandre Sasha Alexandrov, a.s.alexandrov@lboro.ac.uk
}

Received 7 June 2010; Accepted 7 June 2010

Copyright (c) 2010 Alexandre Sasha Alexandrov et al. This is an open access article distributed under the Creative Commons Attribution License, which permits unrestricted use, distribution, and reproduction in any medium, provided the original work is properly cited.

Since the discovery of high-temperature superconductivity in 1986 by George Bednorz and Alex Müller, there has been a huge theoretical effort to understand the mechanism behind it. A lanthanum copper oxide, doped with barium, was the first compound displaying this phenomenon, and now many more high-temperature superconductors based on copper and oxygen have been discovered. These make up the cuprate family of superconductors. More recently fullerenes, $\mathrm{MgB}^{2}$, and in particular iron-based superconductors with high-transition temperatures have been discovered. Cuprates are different from conventional metallic superconductors in that they originate from the charge-doping of parent Mott insulators. The superconductivity arises in weakly coupled doped layers held together by ionic bonding. As well as their high-Tc, they display many unique properties. For instance, they exhibit two different energy scales: a "superconducting" gap (SG) that develops below the superconducting critical temperature which can be seen by extrinsic and intrinsic tunnelling experiments, as well as by high-resolution angleresolved photoemission experiments; and another gap-like feature, the so-called "pseudogap" (PG), that exists in the superconducting state and well above Tc, in the underdoped region with doping smaller than the optimal value at which the maximum Tc occurs. The PG phenomenon was first observed through spin response in underdoped $\mathrm{YBa}_{2} \mathrm{Cu}_{3} \mathrm{O}_{7-\delta}$. Many experiments have since detected this PG, including extrinsic and intrinsic tunnelling; photoemission, pump-probe spectroscopies, calorimetric, and many others. Initially this gap was interpreted as manifestation of preformed electron pairs existing above Tc called small bipolarons, bound together by a strong electron-phonon interaction (EPI). Since then many theoretical explanations have been proposed for the origin of the PG which can roughly be divided into two groups. The first of these groups argues that the PG originates from some competing order, either static or fluctuating, possibly also giving rise to inhomogeneous states. The second group interprets the pseudogap as a precursor of superconductivity and suggests the existence above Tc of preformed pairs with no coherence. Despite intensive research, a microscopic theory capable of describing unusual ARPES and tunnelling data has remained elusive and so the relationship between the SG and PG has remained a debated issue. A detailed and consistent interpretation of the SG, PG, and many other unusual properties could shed light on the key effective interactions in cuprate superconductors. In this regard, the interplay of EPI with correlations could be relevant.

The recently discovered Fe-based high-temperature superconductors (pnictides) also represent a challenging case for the theory of superconductivity. They appear to be rather different from cuprates in terms of their electronic structure; magnetic order, correlation effects, superconducting symmetry; their parent state is metallic rather than insulating. So far, the most popular suggestion for the pairing mechanism has been one that assigns the role of an intermediate boson to spin fluctuations with wave vectors close to $Q=(\pi, \pi)$ (in the Brillouin zone). There are two ways to generate such spin fluctuations: one assumes superexchange between the second neighbours in the Fe lattice and the other exploits the fact that the noninteracting spin susceptibility calculated 
using the electron band structure has a peak, or rather a broad maximum close to $(\pi, \pi)$. A strong argument in favour of the latter scenario is the case of FeSe, where a sister magnetic compound FeTe shows an antiferromagnetic order at a different wave vector $(\pi, 0)$ both in the experiment and in the calculations, but the calculated spin susceptibility still peaks at $Q=(\pi, \pi)$ and the experiment also observes spin fluctuations with the same wave vector. On the other hand, the interplay between spin fluctuations, correlations, and EPI remains a challenging open problem, taking into account controversial isotope effects, structural instabilities, low carrier densities; and some other peculiarities of pnictides.

The absence of consensus on the physics of cuprate superconductors and the recent discovery of iron-based compounds with high-transition temperatures has reemphasized the fundamental importance of understanding the origin of high-temperature superconductivity. Some first principle calculations based on density functional theory (DFT) often predict a rather weak electron-phonon interaction insufficient to explain high transition temperatures. A number of researchers have the opinion that correlations select momentum and frequency windows where EPI is more efficient. Others maintain that the repulsive electron-electron interaction in novel superconductors provides pairing and thus offers high transition temperatures without phonons. On the other hand, some recent studies using numerical techniques cast doubt that simple repulsive models can account for hightemperature superconductivity. Besides, there is substantial experimental evidence that EPI is an important player and needs to be included in a successful theory of high-Tc superconductivity. This was the motivation for the Editors to provide an international forum that would address the intriguing issue of the interplay between strong electronic correlations and sizeable electron-phonon coupling.

This special issue includes both original results and summarizing overviews. The Editors hope that this publication will stimulate the continuing efforts to understand hightemperature and other unconventional superconductors.

\author{
Alexandre Sasha Alexandrov \\ Carlo Di Castro \\ Igor Mazin \\ Dragan Mihailovic
}



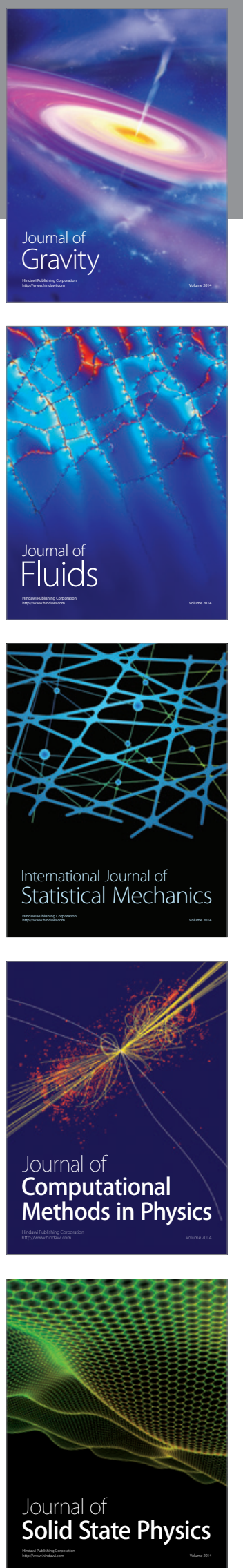

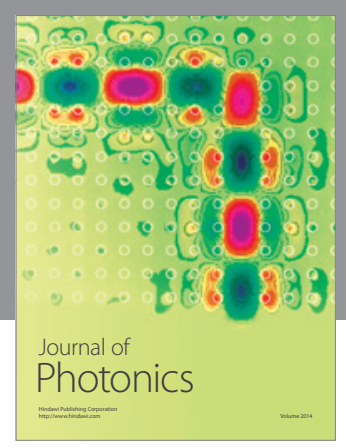

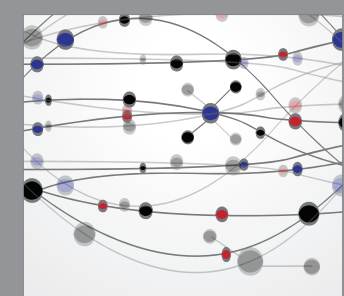

The Scientific World Journal
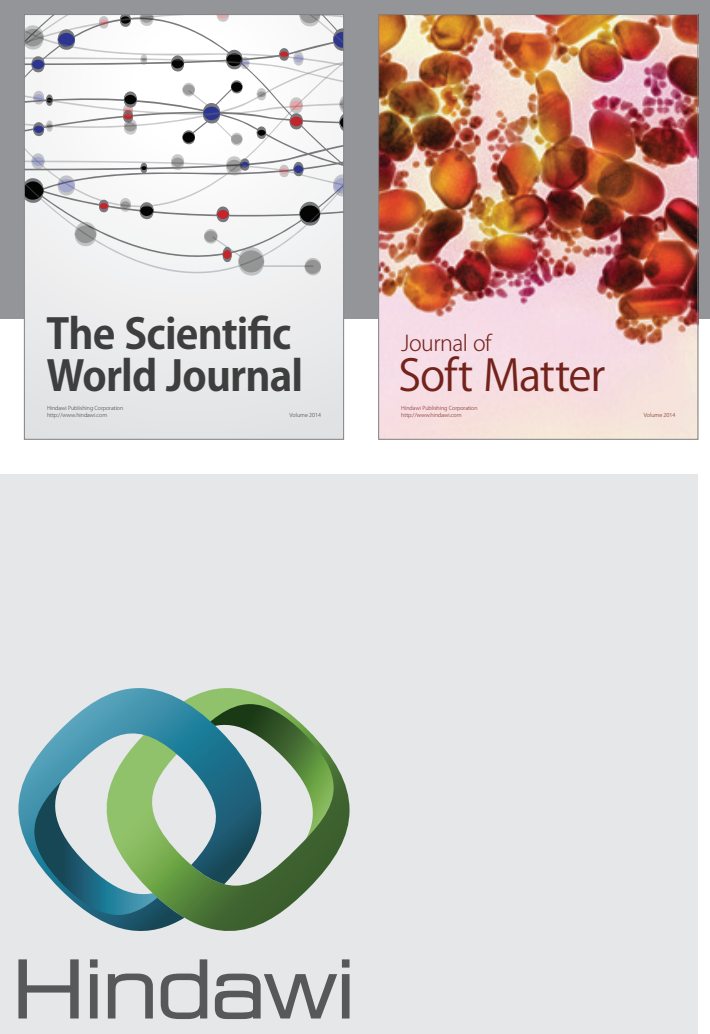

Submit your manuscripts at

http://www.hindawi.com
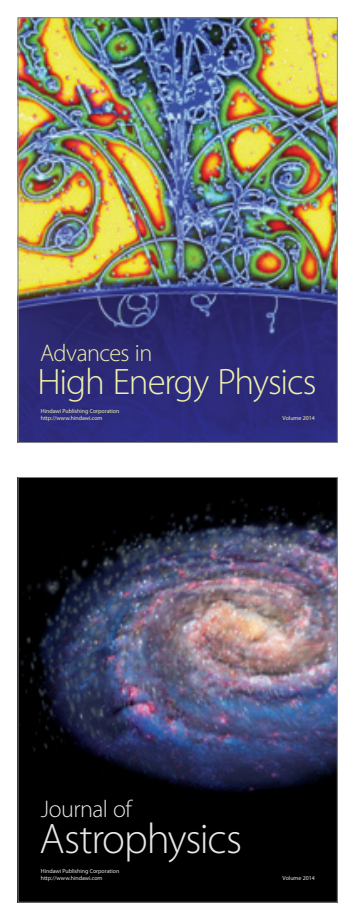
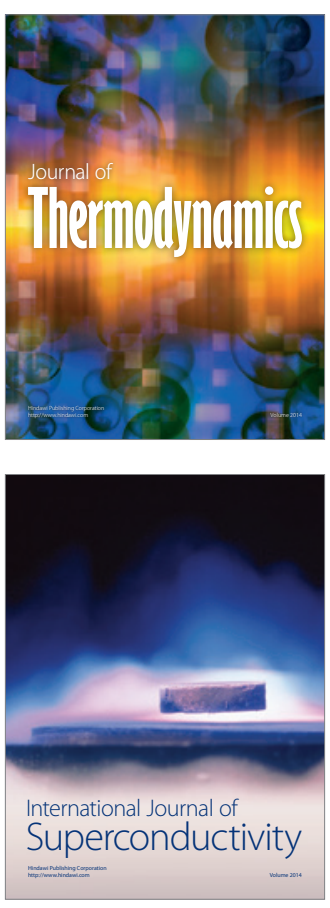
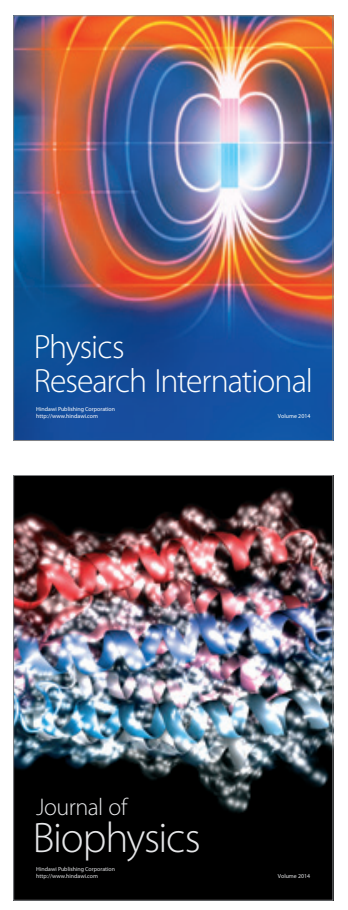
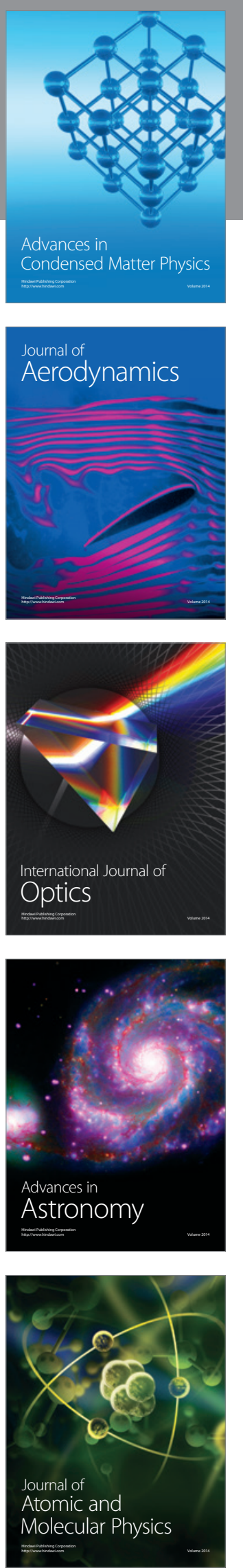